\title{
Magnetically actuated cell-laden microscale hydrogels for probing strain-induced cell responses in three dimensions
}

\author{
Yuhui $\mathrm{Li}^{1,2}$, Guoyou Huang ${ }^{1,2}$, Bin Gao ${ }^{1,2,3}$, Moxiao $\mathrm{Li}^{2,4}$, Guy M Genin ${ }^{1,2,5,6}$, Tian Jian Lu ${ }^{2,4}$ and Feng Xu ${ }^{1,2}$ \\ Living cells respond to their mechanical microenvironments during development, healing, tissue remodeling and homeostasis \\ attainment. However, this mechanosensitivity has not yet been established definitively for cells in three-dimensional (3D) culture \\ environments, in part because of challenges associated with providing uniform and consistent 3D environments that can deliver \\ a large range of physiological and pathophysiological strains to cells. Here, we report microscale magnetically actuated, \\ cell-laden hydrogels ( $\mu$ MACs) for investigating the strain-induced cell response in 3D cultures. $\mu$ MACs provide high-throughput \\ arrays of defined 3D cellular microenvironments that undergo reversible, relatively homogeneous deformation following \\ non-contact actuation under external magnetic fields. We present a technique that not only enables the application of these high \\ strains $(60 \%)$ to cells but also enables simplified microscopy of these specimens under tension. We apply the technique to \\ reveal cellular strain-threshold and saturation behaviors that are substantially different from their 2D analogs, including \\ spreading, proliferation, and differentiation. $\mu \mathrm{MACs}$ offer insights for mechanotransduction and may also provide a view of how \\ cells respond to the extracellular matrix in a $3 \mathrm{D}$ manner.
}

NPG Asia Materials (2016) 8, e238; doi:10.1038/am.2015.148; published online 22 January 2016

\section{INTRODUCTION}

The mechanical environments of cells have a vital role in the development and functionality of living tissues and organs. ${ }^{1-4}$ Cells sense and respond to mechanical cues, such as the mechanical strains and the rigidity of their extracellular matrix (ECM), by adjusting the transmembrane molecules (e.g., integrins) and by reorganizing the intracellular cytoskeleton. ${ }^{5,6}$ Tools for studying strain responses of cells in two-dimensional (2D) environment, including stretchable substrata, ${ }^{7}$ micropost arrays ${ }^{8}$ and $2 \mathrm{D}$ traction microscopy, ${ }^{9}$ are well established. However, observations from these tools are difficult to extend to three-dimensional (3D) culture environments because of fundamental differences in the morphologies and functionalities of cells in $2 \mathrm{D}$ and $3 \mathrm{D}$ cultures. ${ }^{10-12}$ Thus, there is a pressing need for tools to define the mechanical responses of cells in $3 \mathrm{D}$ cultures, and a number of coupled experimental and analytical systems have been developed for this purpose. ${ }^{13-15}$ These mechanobiological tools include ring-like tissue constructs, ${ }^{16}$ tissue constructs adhered to flexible substrata ${ }^{17}$ and $3 \mathrm{D}$ traction microscopy. ${ }^{14}$ However, challenges persist for achieving displacement control, obtaining statistically significant sample sizes, avoiding diffusion barriers for soluble factors and avoiding perturbations introduced by sample handling before mechanical loading.

Recently, magnetically actuated cantilevers have emerged as useful tools for contractility measurements of multicellular tissue constructs. ${ }^{18-20}$ In these systems, 3D microtissues were created and constrained between patterned poly(dimethylsiloxane) cantilevers attached to a magnetically responsive microsphere. Such microtissue force gauges have allowed fast diffusion of soluble factors to multiple samples simultaneously, offered the capability to apply external mechanical loading to the specimens and enabled measurement of the contractility of multicellular tissues under applied force. We previously fabricated magnetic-responsive cell-laden hydrogel fibers and successfully stretched these fibers using external magnetic fields. ${ }^{21}$ Despite these significant advantages, these systems are stable only at low physiological strains and have not been used to successfully characterize cellular mechanobiology at larger physiological strains for skeletal and cardiac muscle, which can be $>20 \% .^{22,23}$ Characterizing the entire range of physiological strains is crucial for understanding injury-level mechanobiology, soft-tissue healing, tissue remodeling and homeostasis attainment. ${ }^{24-26}$ Additionally, none of the above methods

\footnotetext{
${ }^{1}$ The Key Laboratory of Biomedical Information Engineering of Ministry of Education, School of Life Science and Technology, Xi'an Jiaotong University, Xi'an, China; ${ }^{2}$ Bioinspired Engineering and Biomechanics Center (BEBC), Xi'an Jiaotong University, Xi'an, China; ${ }^{3}$ Institute of Digestive Disease, Xijing Hospital, Fourth Military Medical University, Xi'an, China; ${ }^{4}$ State Key Laboratory for Strength and Vibration of Mechanical Structures, School of Aerospace, Xi'an Jiaotong University, Xi'an, China; ${ }^{5}$ Department of Biochemistry and Molecular Biophysics, Washington University School of Medicine, Saint Louis, MO, USA and ${ }^{6}$ Department of Mechanical Engineering and Materials Science, Washington University, Saint Louis, MO, USA

Correspondence: Dr G Huang or Professor F Xu, The Key Laboratory of Biomedical Information Engineering of Ministry of Education, School of Life Science and Technology, Xi'an Jiaotong University, No. 28, Xianning West Road, Xi'an, Shaanxi, 710049, China.
}

E-mail: wwgyhuang@mail.xjtu.edu.cn or fengxu@mail.xjtu.edu.cn

Received 13 August 2015; revised 25 October 2015; accepted 17 November 2015 
has yet been able to determine the independent effects of strain and stiffness on cell behavior, which, in 3D, are not independent.

To address these challenges, we developed defined 3D cellular microenvironments called microscale magnetically actuated, cell-laden hydrogels ( $\mu \mathrm{MACs}$ ) using a multiple-photolithography method. $\mathrm{MACs}$ can undergo reversible, relatively homogeneous strains up to $60 \%$ through non-contact, focused magnetic actuation. Herein, we describe the $\mu \mathrm{MAC}$ system and its application to the study of strain-induced spreading, proliferation and differentiation of cells in 3D cultures. We found that spreading and proliferation of NIH-3T3 fibroblasts were significantly enhanced by strains up to an asymptotic saturation level of $40-60 \%$. The differentiation and maturation of C2C12 myofibers within $\mu$ MACs also occurred after a sustained strain level of $\sim 40 \%$ was reached. The $\mu$ MAC system enabled delineation of strain-induced cell responses from stiffness-induced cell responses in $3 \mathrm{D}$ hydrogels while simultaneously enabling simple visualization in real time over the objective of a confocal fluorescence microscope.

\section{MATERIALS AND METHODS}

\section{$\mathrm{Fe}_{3} \mathrm{O}_{4}$ nanoparticle and GelMA synthesis}

$\mathrm{Fe}_{3} \mathrm{O}_{4}$ nanoparticles were synthesized via co-precipitation. ${ }^{27}$ First, $1 \mathrm{M}$ $\mathrm{FeCl}_{3} \cdot 6 \mathrm{H}_{2} \mathrm{O}$ and $2 \mathrm{M} \mathrm{FeCl} \cdot 4 \mathrm{H}_{2} \mathrm{O}$ solutions were prepared by dissolving iron salts in $2 \mathrm{M} \mathrm{HCl}$ solutions. Later, $10 \mathrm{ml}$ of $1 \mathrm{M} \mathrm{FeCl}_{3}$ solution was mixed with $2.5 \mathrm{ml}$ of $2 \mathrm{M} \mathrm{FeCl}_{2}$ solution in a flask. This solution was stirred, followed by the slow addition of $21 \mathrm{ml}$ of $1.5 \mathrm{M} \mathrm{NaOH}$ solution, until a pH of 13 was reached. The precipitated powder was isolated using a permanent magnet and then dispersed in deionized water for future use. Gelatin methacrylate (GelMA) was synthesized as described previously. ${ }^{28}$ At a temperature of $65^{\circ} \mathrm{C}$, type A porcine skin gelatin powder (Sigma-Aldrich, St Louis, MO, USA) was added to Dulbecco's phosphate-buffered saline solution (DPBS; Gibco, Carlsbad, CA, USA) and stirred until fully dissolved to obtain a concentration of $10 \%\left(\mathrm{w} \mathrm{v}^{-1}\right)$. Methacrylic anhydride (Sigma-Aldrich), a photocrosslinkable monomer, ${ }^{29}$ was added to this solution at a rate of $0.5 \mathrm{ml} \mathrm{min}^{-1}$ while stirring at $50{ }^{\circ} \mathrm{C}$ until the target volume was reached. The mixture was then dialyzed against distilled water at $40^{\circ} \mathrm{C}$ for 1 week using $12-14 \mathrm{kDa}$ cutoff dialysis tubing (Spectrum Labs, Rancho Dominguez, CA, USA). The water was changed every day to remove salts and methacrylic acid. After that, the GelMA solution was lyophilized and stored at $-80^{\circ} \mathrm{C}$ for future use.

\section{Nanoparticle characterization}

Transmission electron microscopy images were acquired at an accelerating voltage of $100 \mathrm{kV}$ using a Hitachi HT7700 (Tokyo, Japan) microscope. X-ray diffraction data were collected using a X-ray diffraction-7000 diffractometer (Shimadzu, Kyoto, Japan) with $\mathrm{Cu} \mathrm{K}_{\alpha}$ radiation. Particle size was determined using the Scherrer method, and magnetization of particles was carried out at room temperature using a Lakeshore 6653 magnetometer (Lakeshore, Westerville, OH, USA).

\section{Fabrication of $\mu$ MACs}

A multiple-step photolithography method was developed to fabricate $\mu$ MACs. A two-part poly(methyl methacrylate) mold composed of (i) a cover mold combined with a photomask and (ii) a base mold combined with two layers of PET (poly(ethylene) terephthalate; 3M, St Paul, MN, USA) (thickness in $150 \mu \mathrm{m}$ ) was designed. A PET membrane was used to form a container in the base mold. Three types of masks were also designed (Supplementary Figure S1).

First, the magnetically actuated layer was fabricated. $\mathrm{An}^{\mathrm{F}} \mathrm{F}_{3} \mathrm{O}_{4}$ nanoparticle solution at a concentration of $5 \%\left(\mathrm{w} \mathrm{v}^{-1}\right)$ was mixed with a $15 \%\left(\mathrm{w} \mathrm{v}^{-1}\right)$ PEGDMA (poly(ethylene glycol) dimethacrylate) precursor solution (MW $=1000$ Da; Polysciences, Warrington, PA, USA) and 0.05\% $\left(\mathrm{w} \mathrm{v}^{-1}\right)$ photoinitiator (2-hydroxy-2-methylpropiophenone; TCI, Shanghai, China). Then, $500 \mu \mathrm{l}$ of this mixture was pipetted into the PET container and covered with a glass slide. The PEGDMA solution was then crosslinked by exposing to $365 \mathrm{~nm}$ UV light at a power of $2.9 \mathrm{~mW} \mathrm{~cm}^{-2}$ for $45 \mathrm{~s}$. Next, the constrained layer was fabricated by exposing a $20 \%\left(\mathrm{w} \mathrm{v}^{-1}\right)$ PEGDMA precursor solution mixed with $0.05 \%\left(\mathrm{w} \mathrm{v}^{-1}\right)$ photoinitiator to UV light for $60 \mathrm{~s}$. Finally, a population of cells mixed with GelMA solution and $0.05 \%\left(\mathrm{w} \mathrm{v}^{-1}\right)$ photoinitiator was pipetted onto the crosslinked magnetically actuated and constrained layer, soaked for $10 \mathrm{~min}$ and then exposed to UV light for $30 \mathrm{~s}$, which has been demonstrated not to affect cell viability or long-term cell health. ${ }^{30}$ To quantify cell viability and differentiation, an encapsulated cell density of $1 \times 10^{5}$ cells $\mathrm{ml}^{-1}$ was used. To quantify cell proliferation and spreading, an encapsulated cell density of $1 \times 10^{4}$ cells $\mathrm{ml}^{-1}$ was used.

\section{Magnetic characterization, actuation and simulation}

The external magnetic field was calibrated using an $\mathrm{NdFeB}$ permanent magnet (40 mm $\times 20 \mathrm{~mm} \times 10 \mathrm{~mm}$ ) (K\&J Magnetics, Pipersville, PA, USA). Movement of the magnet was controlled by a displacement stage. For all cell experiments, magnetic loading began after $6 \mathrm{~h}$ of encapsulation. Magnetic loading was applied to each sample $10 \mathrm{~h}$ per day. A series of numerical simulations using commercially available software (COMSOL Multiphysics 4.0a; COMSOL, Stockholm, Sweden) was performed to characterize the magnetic field distribution and force generation. In these simulations, the magnetic force applied to a GelMA layer was modeled as a uniform pressure on the surface closest to the magnet; in-plane displacements were constrained to be zero on that surface, and all displacements were constrained to be zero on the opposite surface (the one farthest from the magnet). These boundary conditions were appropriate given the difference in stiffness between the PEGDMA and GelMA layers. GelMA was treated as an incompressible, isotropic Neo-Hookean solid with an elastic modulus derived from the experimental work described below. To calibrate the magnetic force applied to the magnetically actuated layer, a standard Stokes drag assay was used. A PEGDMA hydrogel sphere (diameter of $8 \mathrm{~mm}$ ) encapsulating $5 \%\left(\mathrm{w} \mathrm{v}^{-1}\right)$ magnetic nanoparticles (MNPs) was placed in a poly(ethylene oxide) solution of defined viscosity (Sigma-Aldrich), after which a magnetic field was applied. The speed of the magnetic sphere was estimated as a function of separation between the end of the magnetic field focuser and the center of the sphere using a video taken with a $\times 20$ objective on a high-speed camera (Phatom Cinestream v.711; Vision Research, Wayne, NJ, USA) at 1000 images per second. The applied force on the magnetically actuated layer was then estimated using

$$
\vec{F}_{\text {magnetic }}=6 \pi R v \vec{U}+m \vec{a}
$$

where the first term on the right side represents the viscous drag force in which $\nu$ is the dynamic viscosity of the poly(ethylene oxide) solution, $R$ is the radius of the hydrogel sphere and $\vec{U}$ is the speed of the hydrogel sphere; and the second term on the right side represents the inertial force in which $m$ is the mass of the hydrogel sphere and $\vec{a}$ is its acceleration.

Cell viability, proliferation and spreading volume characterization To measure cell viability, GelMA layers encapsulated with NIH-3T3 fibroblasts were stained using a live-dead assay (Molecular Probes, Carlsbad, CA, USA) following the manufacturer's instructions. GelMA layers were resected from the $\mu \mathrm{MACs}$ and incubated in a solution of $2 \mu \mathrm{g} \mathrm{m} l^{-1}$ calcein AM and $5 \mu \mathrm{g} \mathrm{m}^{-1}$ propidium iodide at $37^{\circ} \mathrm{C}$ for $30 \mathrm{~min}$. Stained cells were counted from these images using Image-Pro Plus (Media Cybernetics, Silver Spring, MD, USA). Fibroblast proliferation in GelMA layers was characterized using a Cell Counting Kit-8 (Molecular Probes). To measure the cell spreading volume, F-actin stress fibers and nuclei of cells were stained with rhodamine-conjugated phalloidin (Cytoskeleton, Denver, CO, USA) and DAPI (4',6-diamidino-2phenylindole), respectively. For stress fiber staining, cells in the synthetic tissue slices were fixed using $4 \%$ formaldehyde (Sigma-Aldrich) for $15 \mathrm{~min}$, permeabilized with $0.5 \%$ Triton X-100 (Sigma-Aldrich) for $5 \mathrm{~min}$ and then incubated with $200 \mu \mathrm{l}$ of $100 \mathrm{~nm}$ rhodamine phalloidin solution in the dark at room temperature for $40 \mathrm{~min}$. For nuclear staining, cells were counterstained with $200 \mu \mathrm{l}$ of $100 \mathrm{~nm}$ DAPI in DPBS for $5 \mathrm{~min}$. Images were captured using a confocal microscope (Olympus, Tokyo, Japan). The cell spreading area was analyzed from 3D reconstructions of confocal image slices using Imaris software (Bitplane, Switzerland). 


\section{Cell differentiation characterization}

After culture and mechanical treatment, $\mathrm{C} 2 \mathrm{C} 12$ myotubes in the GelMA layers were fixed using $4 \%$ formaldehyde for $20 \mathrm{~min}$, followed by three washes with DPBS. The permeabilization step was performed using $0.5 \%$ Triton X-100 for $10 \mathrm{~min}$ at room temperature. The cells were then exposed to $5 \%$ bovine serum albumin dissolved in DPBS for $15 \mathrm{~min}$. Two primary mouse monoclonal immunoglobulin G antibodies against myosin heavy chain (МyHC) (ab-7784; Abcam, Cambridge, UK) and sarcomeric $\alpha$-actinin (ab-72592, Abcam) were added to the sample at a dilution of 1:1000 in DPBS, and the samples were incubated at $4{ }^{\circ} \mathrm{C}$ overnight. The sample was then washed three times with DPBS, treated with a goat anti-mouse AlexaFluor 594 antibody (ab-150080 against MyHC; Abcam) and goat anti-mouse AlexaFluor 488 antibody (ab-150113 against sarcomeric $\alpha$-actinin; Abcam) at a dilution of 1:1000 in DPBS, and incubated at $37^{\circ} \mathrm{C}$ for $1 \mathrm{~h}$. The nuclei were stained according to the protocol described above. The stained $\mathrm{C} 2 \mathrm{C} 12$ myotubes were then imaged and reconstructed using confocal microscopy.

\section{Western blot analysis}

Cells or myotubes were isolated using RIPA lysis and an extraction buffer with a protease inhibitor (Beyotime, Shanghai, China). Protein concentration was determined using a BCA Protein Assay Kit (Pierce of Thermo Scientific, Rockford, IL, USA) according to the manufacturer's protocols. Each protein sample was applied to sodium dodecyl sulphate-polyacrylamide gel electrophoresis gels, transferred to pure nitrocellulose membranes (Perkin-Elmer Life Sciences, Boston, MA, USA), and blocked with 5\% nonfat milk. The membranes were incubated overnight with the primary antibody at $4{ }^{\circ} \mathrm{C}$. Then, the membranes were incubated with anti-rabbit or anti-mouse secondary antibodies at room temperature for $1 \mathrm{~h}$. Chemiluminescent detection was performed using an ECL Western Blotting Detection Kit (Thermo Fisher, Rockford, IL, USA). Images were analyzed using Quantity One software (Bio-Rad, Shanghai, China).

\section{Real-time PCR}

Total RNA was extracted from the cells and purified following the manufacturer's protocol (DNA/RNA Protein Isolation Kit (DP423) (Tiangen Biotech, Beijing, China)). Reverse transcription was performed using a PrimeScript RT-PCR Kit (Takara, Dalian, China) followed by semiquantitative realtime PCR using specific primers. The primer sequences were as follows: myogenin (forward: 5'-GAGACATGAGTGCCCTGACC-3' and reverse: 5'AGGCTTTGGAACCGGATAGC-3'); Mrf4 (forward: $5^{\prime}$-CGAAAGGAGGA

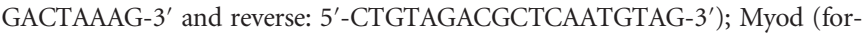
ward: $5^{\prime}$-CCAGGACACGACTGCTTTCT- $3^{\prime}$ and reverse: $5^{\prime}$-TCTGGTGAGT CGAAACACGG-3'); and MyHC IId/x (forward: 5'-GCGACAGACACCTCC TTCAAG- $3^{\prime}$ and reverse: $5^{\prime}$-TCCAGCCAGCCAGCGATG-3'). The target gene expression was normalized to the GAPDH (glyceraldehyde-3-phosphate dehydrogenase) gene expression. ${ }^{31}$ Real-time PCR was performed using a 7500 Fast Real-Time PCR System (Life Technologies, Paisley, UK) with $0.8 \mu \mathrm{l}$ of cDNA, $0.4 \mu \mathrm{l}$ of the primer set, $3.8 \mu \mathrm{l}$ of $\mathrm{ddH}_{2} \mathrm{O}$ and $5 \mu \mathrm{l}$ of SYBR Green (Takara, Dalian, China). After an initial denaturation step at $95^{\circ} \mathrm{C}$ for $30 \mathrm{~s}$, real-time PCR was performed over 45 cycles at $95^{\circ} \mathrm{C}$ for $5 \mathrm{~s}$ and $60{ }^{\circ} \mathrm{C}$ for $30 \mathrm{~s}$, followed by a melting curve analysis. Gene expression analysis was repeated at least three times for each sample.

\section{Statistical analysis}

All data collected are presented as the mean \pm s.d. of 10-15 samples. All data were performed with analysis of variance with Tukey's post hoc test or paired $t$-test. A $P$-value of 0.05 or less was considered statistically significant.

\section{RESULTS AND DISCUSSION}

Brush-shaped $\mu \mathrm{MACs}$ were fabricated based on a multiple-step photolithography approach (Figures 1a-c). Each $\mu$ MAC sample contained (i) a stiffness-tunable cell-laden hydrogel layer of GelMA $(500 \mu \mathrm{m} \times 900 \mu \mathrm{m}, 130 \mu \mathrm{m}$ total thickness) (Supplementary Figure S1), which is a biocompatible and photocrosslinkable hydrogel derived from natural gelatin that is effective for $3 \mathrm{D}$ cell encapsulation; ${ }^{28,32}$ and (ii) two stiff and strong hydrogel layers of PEGDMA, including a 'magnetically actuated' layer with encapsulated MNPs and a PEGDMA base layer without MNPs (Figures 1d and e). PEGDMA proved effective as a material for the actuated layer because it has good mechanical properties, high pattern fidelity and high resolution. ${ }^{33}$ The PEGDMA fraction in these layers was optimized to avoid inducing strain in the magnetically actuated layers during mechanical loading of the specimens (Supplementary Figure S2). Interfacial failure between the GelMA and PEGDMA layers was ameliorated by toughening using the double-network principle, ${ }^{34}$ and differential swelling of the two layers was overcome by tuning the GelMA fraction (Supplementary Figure S3). The swelling ratio of the PEGDMA layer (with and without MNPs) was relatively constant over the relevant concentration range of $10-20 \%\left(\mathrm{w} \mathrm{v}^{-1}\right)$ (Supplementary Figure S3a). A similar swelling ratio could also be achieved using $10 \%$ $\left(\mathrm{w} \mathrm{v}^{-1}\right)$ GelMA.

To apply non-contact mechanical actuation to $\mu \mathrm{MACs}$, in-house synthesized $\mathrm{Fe}_{3} \mathrm{O}_{4}$ MNPs were encapsulated into the PEGDMA layer via a simple blending method, as demonstrated previously (Figure 2a). ${ }^{35}$ The structure and morphology of MNPs, characterized by X-ray diffraction and transmission electron microscopy, showed that the MNPs were uniform in size $(20 \mathrm{~nm})$ (Supplementary Figure S4 and Figure 2b). The superparamagnetic behavior of the synthesized MNPs was assessed through hysteresis loops measured using a vibrating sample magnetometer (Figure 2c). The magnetization saturation $\left(M_{\text {sat }}\right)$ was $75 \mathrm{emu} \mathrm{g}^{-1}$, which is suitable for tensile straining of $\mu \mathrm{MACs}$. The force applied to the magnetically actuated layer within the $\mu \mathrm{MACs}$ was approximated using finite element simulations that accounted for factors including the volume of MNPs, the magnetic field gradients and the separation of the MNPs from the permanent magnets (Supplementary Figure S5). The combined force on the particle ensembles can be estimated according to

$$
\vec{F}=\sum V(\vec{x}) \mu_{0}^{-1} M_{\text {sat }} \vec{\nabla} \vec{B}(\vec{x})
$$

where $V(\vec{x})$ is an estimate of the volume of the nanoparticle clusters at position $\vec{x}, \mu_{0}$ is the magnetic permeability of a vacuum, $M_{\text {sat }}$ is the saturation moment of an individual nanoparticle and $\vec{\nabla} \vec{B}(\vec{x})$ is the external magnetic field gradient tensor at position $\vec{x}$. A Stokes drag assay used to calibrate the magnetic force applied to the MNPs in the magnetically actuated layers ${ }^{36}$ showed that the relationship between force and separation from the permanent magnet was approximately an inverse cubic, which matches the simulation result (Supplementary Figure S6a). The stress-strain curves measured for the $\mu$ MACs indicated a nearly linear response up to exceptionally high levels of strain (up to 60\%) (Figure $2 \mathrm{~d}$ and e). The responses of the cell-laden hydrogel layers varied with the GelMA fraction but were linear and repeatable for all cases, suggesting that the stiffness of the GelMA layer did not change in response to strains in the range of 0-60\%. Finite element simulations indicated that the strain fields within the $\mu \mathrm{MACs}$ were uniform within a few percent, except near the free edges of the interface between the PEGDMA and the GelMA layers, where strain and stress concentrations were evident (Figure 2f). The elastic moduli of the cell-laden hydrogel layers, reported as the slopes of these stress-strain curves, were $6 \mathrm{kPa}$ for $10 \%\left(\mathrm{w} \mathrm{v}^{-1}\right)$, $10 \mathrm{kPa}$ for $15 \%\left(\mathrm{w} \mathrm{v}^{-1}\right)$ and $20 \mathrm{kPa}$ for $20 \%\left(\mathrm{w} \mathrm{v}^{-1}\right)$ GelMA (Supplementary Figure S6b).

Real-time observations of the deformation of the $\mu$ MACs under tension were achieved using a simple loading device (Supplementary Figure S7). This system enabled simple visualization of the hydrogel samples in real time through the objective of a fluorescence 
a

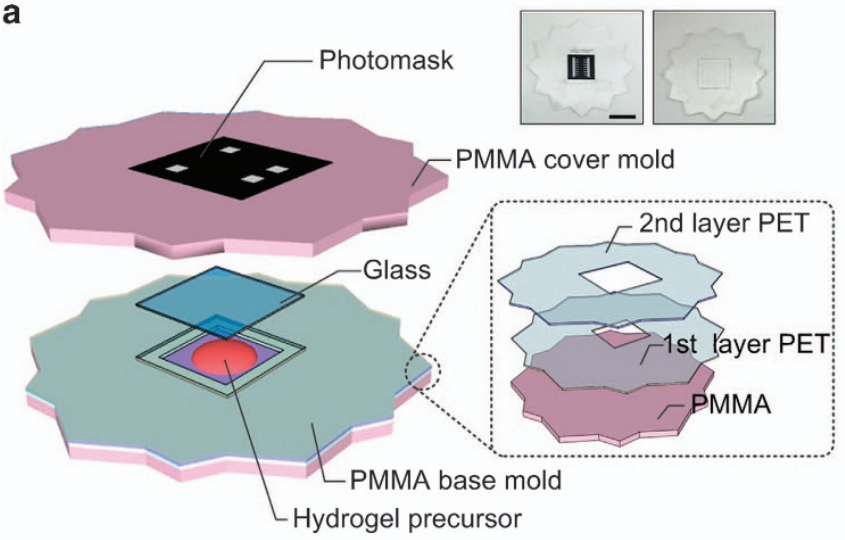

Chip design

C
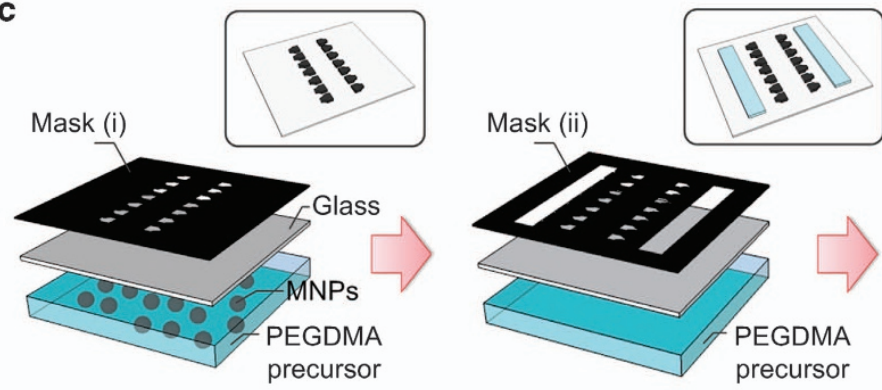

b

UV crosslinking

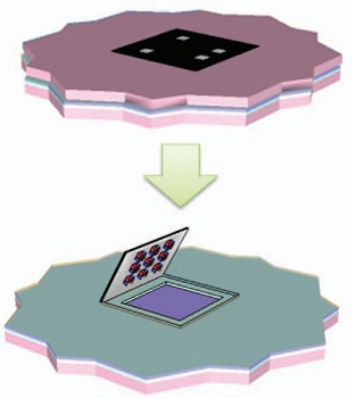

Photolithography

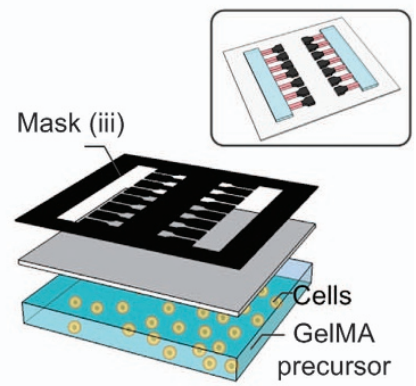

Multiple-step photolithography strategy

d

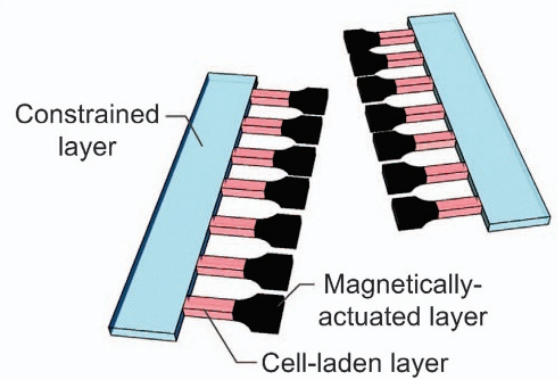

e

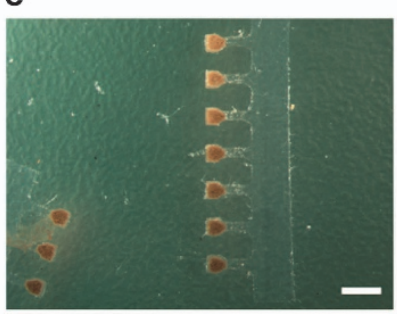

Figure 1 (a and b) A poly(methyl methacrylate) (PMMA) mold composed of cover and base parts was used to fabricate microscale magnetically actuated, cell-laden hydrogels ( $\mu$ MACs). The hydrogel precursor solution was pipetted into the spacer $(24 \mathrm{~mm} \times 0.15 \mathrm{~mm})$ in the base mold and exposed to $2.9 \mathrm{~mW} \mathrm{~cm}^{-2}$ of UV light. (c) Three types of masks were designed, one for generation of each of the layers (schematics and optical images in $\mathbf{d}$ and $\mathbf{e}$ ): (i) a 'magnetically actuated' PEGDMA (poly(ethylene glycol) dimethacrylate) layer encapsulating magnetic nanoparticles (MNPs), (ii) a gelatin methacrylate (GeIMA) layer encapsulating cells to be tested and (iii) a stiff, constrained layer. Scale bars: (a) $2 \mathrm{~cm}$ and (e) $2 \mathrm{~mm}$. PET, poly(ethylene) terephthalate; PMMA, poly(methyl methacrylate); UV, ultraviolet.

microscope and is the first high-throughput device capable of doing so. ${ }^{37}$ The tensile tests of the $\mu$ MACs were performed in situ with varied high strain levels, thus overcoming the challenges faced by existing 3D culture systems such as sample handling, diffusion barriers, specimen variability and limited strain levels induced by mechanical loading.

This definable, linear stress-strain relationship (Figure 2c) enabled us to independently probe the effects of ECM stiffness and strain on cell behaviors in a 3D microenvironment. We first studied the cell viability in $\mu \mathrm{MACs}$ with different stiffnesses $(6,10$ and $20 \mathrm{kPa})$ using Live-Dead assays. As reported in Figures $3 \mathrm{a}$ and $\mathrm{b}$ and Supplementary Figure S8, $>90 \%$ of the NIH-3T3 fibroblasts remained viable after encapsulation for $24 \mathrm{~h}$ in three types of $\mu \mathrm{MACs}$. However, the number of dead cells significantly increased in the $\mu$ MACs with stiffnesses of 10 and $20 \mathrm{kPa}$ after culture for 3 days, indicating that cell viability is strongly dependent on $\mu \mathrm{MAC}$ stiffness. General losses of cell viability in stiffer hydrogels (higher monomer concentration) occurred possibly because of limited pore size, encapsulation stress and nutrient limitations, all of which have been previously demonstrated. ${ }^{38,39}$ The NIH-3T3 fibroblasts encapsulated in the $\mu$ MACs with a stiffness of $6 \mathrm{kPa}$ showed high viability even after 5 days of culture (Figure $3 \mathrm{~b}$ ). Note that the $\mu$ MACs with a stiffness of $6 \mathrm{kPa}$ with $10 \%\left(\mathrm{wv}^{-1}\right)$ GelMA had the highest tolerance to strain and were used in subsequent studies. 3D reconstructions of confocal slices of fibroblasts in the strained $\mu \mathrm{MACs}$ revealed the enhancement of cell spreading in response to straining (Figures $3 \mathrm{c}$ and $\mathrm{d}$ ), as reflected by the increase in mean cell volume. In unstrained $\mu \mathrm{MACs}$, cells had spherical morphologies with an average cell volume of $3100 \pm 150 \mu \mathrm{m}^{3}$ per cell. This volume increased rapidly to an asymptotic saturation level 
a

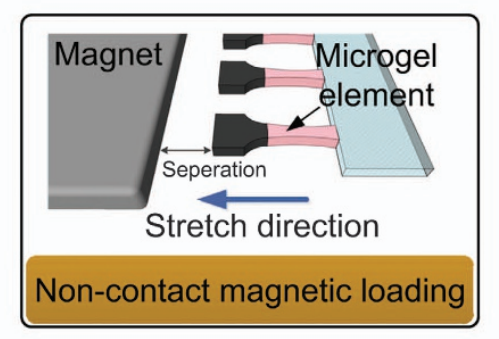

b

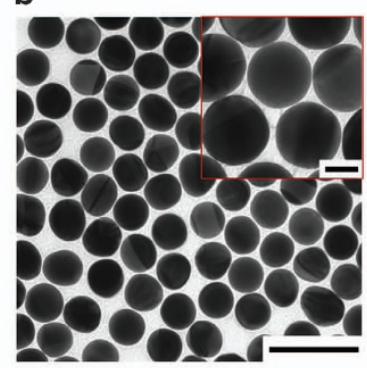

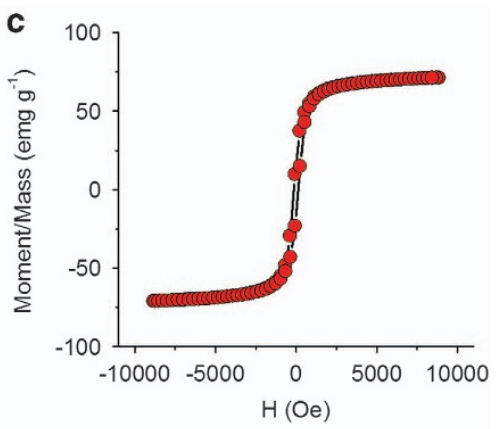

e

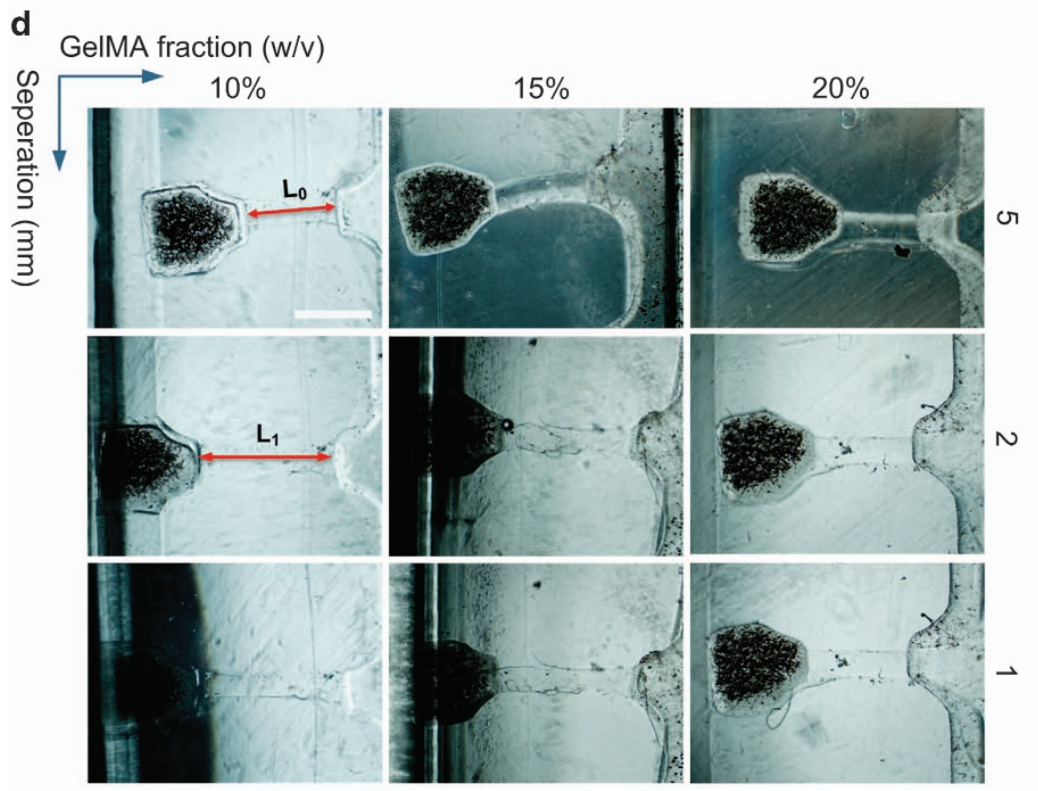

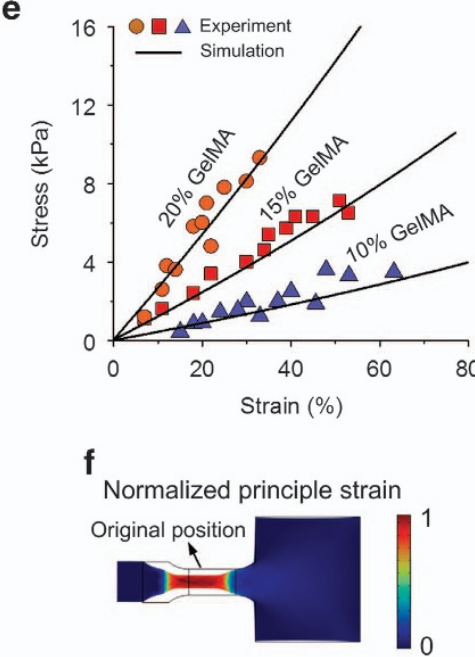

Normalized principle stress

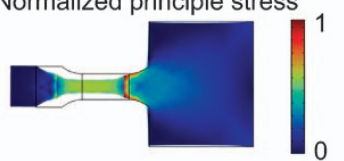

Figure 2 (a) Schematic of non-contact and magnetically actuated loading methods for microscale magnetically actuated, cell-laden hydrogels ( $\mu$ MACs). The movement of the permanent magnet was controlled using a linear stage. (b) Representative transmission electron microscopy image of synthesized $\mathrm{Fe}_{3} \mathrm{O}_{4}$ nanoparticles. (c) Magnetization of $\mathrm{Fe}_{3} \mathrm{O}_{4}$ nanoparticles. The magnetic moment per unit mass showed very little hysteresis. From these measurements, the saturation moment was estimated as $M_{\text {sat }}=75 \mathrm{emug}^{-1}$. (d) Representative images of magnetic stretching of $\mu$ MACs with varying gelatin methacrylate (GeIMA) content and degrees of separation from the applied magnet. (e) Stress-strain curves for cell-laden layers composed of different GelMA fractions were highly linear (symbols: experiment involving straining to failure; lines: simulation). (f) Simulated strain (left) and stress (right) distributions in GelMA layers under magnetic actuation showed highly uniform mechanical fields. Contours were normalized to the peak values. GelMA modulus: $6 \mathrm{kPa}$. Scale bars: (b) $50 \mathrm{~nm}$ and $10 \mathrm{~nm}$ (inset), and (d) $1 \mathrm{~mm}$.

(14 $000 \pm 200 \mu \mathrm{m}^{3}$ per cell) at higher strains (40-60\%) after culture for 3 days. The external magnetic fields showed no statistically significant effects on cellular responses independent of the mechanical strains they actuated (Supplementary Figures S9 and S10). To monitor cell numbers in strained and unstrained $\mu \mathrm{MACs}$, a Cell Counting Kit- 8 was used. The results showed that $\mu$ MACs strained up to $40 \%$ can significantly enhance cell proliferation after 1 day of culturing. In all of the $\mu \mathrm{MACs}$ tested, strain activation was required for substantial proliferation of cells, indicating that cell proliferation is strongly related to strain. These phenomena can be explained only through active cellular processes. The increased spreading area could be explained by the passive stretching of cells in which higher passive strains simply increase the volume available for cells to occupy. The extent of cell proliferation also increased with the degree of straining of the $\mu \mathrm{MACs}$ with peak proliferation at $\sim 40 \%$ strain (Figure $3 \mathrm{e}$ ). Fibroblasts cultured in $2 \mathrm{D}$ require $1-2 \mathrm{~h}$ to reach steady-state proliferation, but this time depends strongly upon mechanical factors, specifically myosin-II-modulated contractility. The absence of any such effect on the strained matrix suggests that a higher strain affects the membrane differently than actomyosin contractility. In all cases, the consistency of the spreading and the proliferation time suggests that the spreading and the proliferation rates are limited in 3D because of active processes within the cells, but further work is required to determine the sources of these effects and their pathways.

To demonstrate the utility of the large strain $\mu$ MAC platform in understanding tissue development, the $\mathrm{C} 2 \mathrm{C} 12$ myoblast cell line was studied for evidence of myotube differentiation, a key step in the myogenesis process, in 3D culture. This is of technological and therapeutic importance because a 3D model of myotubes holds potential for engineering functional skeletal muscle tissues as both tissue substitutes and models for muscle physiology, and for utilization as soft actuators for a variety of biohybrid devices. The $\mu \mathrm{MAC}$ platform was successful in producing dense, aligned and mature myotubes. A critical level of straining was required for extensive 
a
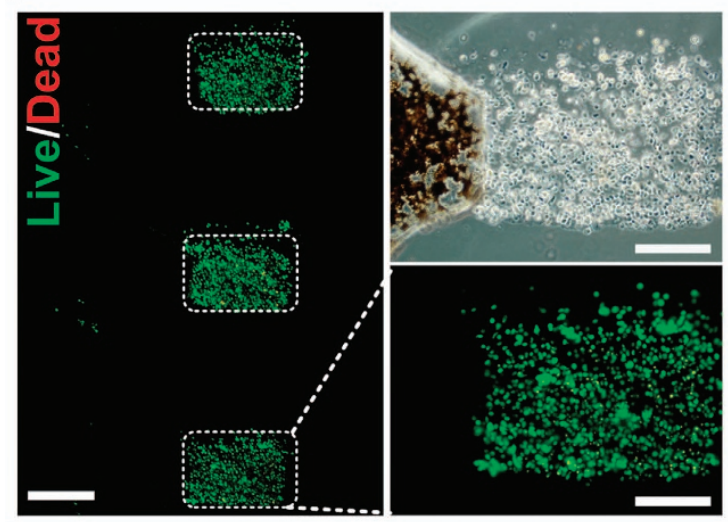

b

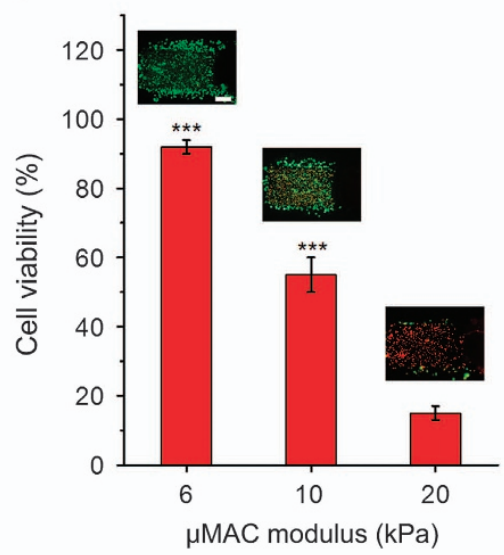

C
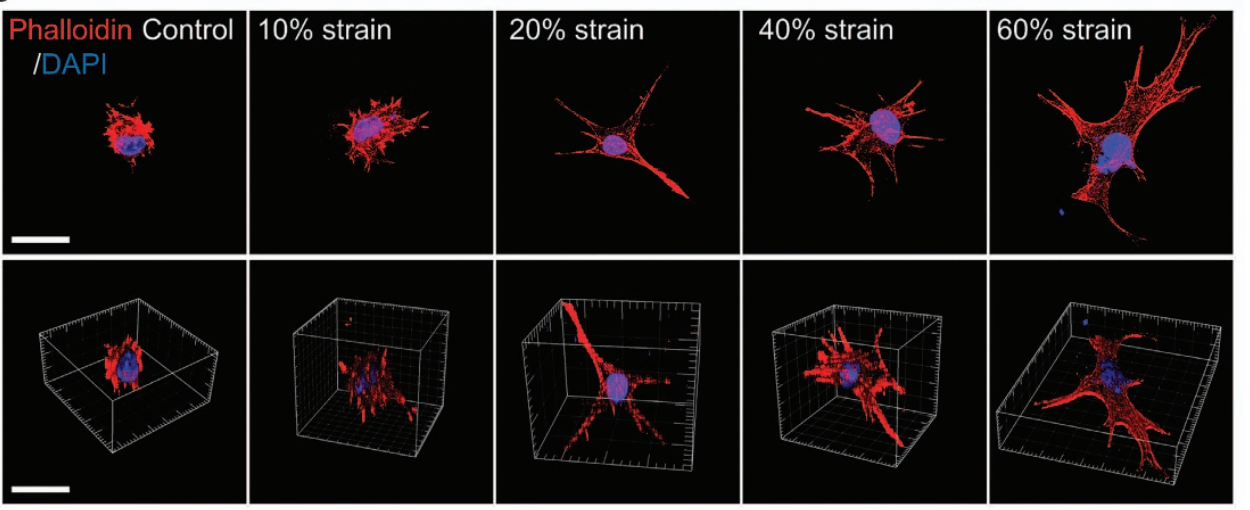

d
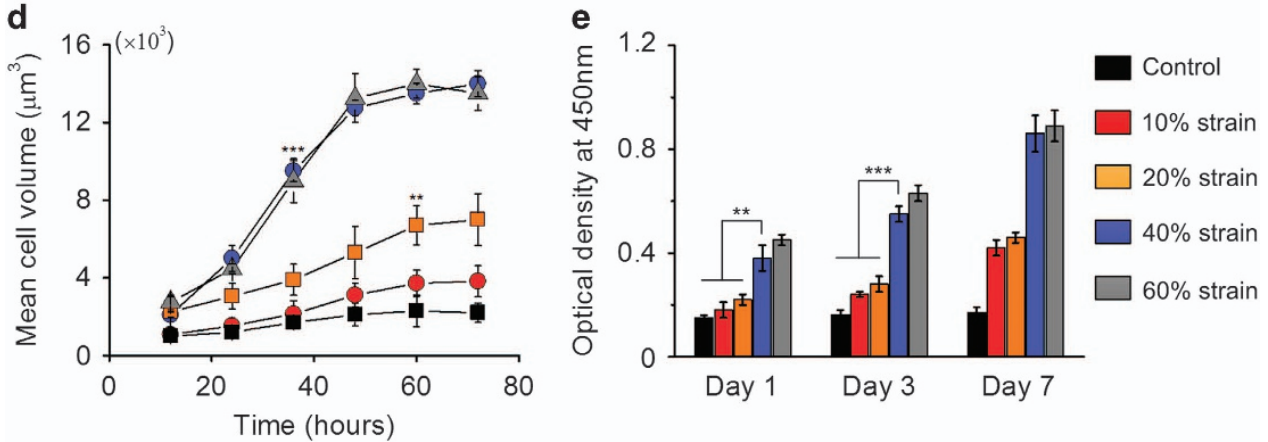

Figure 3 (a) Phase-contrast and live-dead fluorescence images of NIH-3T3 fibroblasts in unstrained microscale magnetically actuated, cell-laden hydrogels ( $\mu$ MACs) after 1 day of encapsulation. Gelatin methacrylate (GeIMA) modulus: $6 \mathrm{kPa}$. (b) Live-dead fluorescence images revealed viability of encapsulated fibroblasts in cell-laden layers with a range of GelMA modulus (6,10 and $20 \mathrm{kPa}$ ) after 5 days of culture. (c) Fluorescent confocal images (top) and threedimensional (3D) reconstruction (bottom) of encapsulated fibroblasts in $\mu \mathrm{MACs}$ (modulus: $6 \mathrm{kPa}$ ) under different strain conditions. Cellular F-actin fibers were stained with phalloidin (red), and nuclei were stained with DAPI (4',6-diamidino-2-phenylindole) (blue). (d) The mean cell spreading volume increased with culture time and increased strain levels, rising rapidly to an asymptotic saturation level at a critical strain in the range of 40-60\%. (e) Cell proliferation increased with time and with strain levels, saturating at $~ 40 \%$ strain. Scale bars: (a) $500 \mu \mathrm{m}$ (left) and $200 \mu \mathrm{m}$ (right), (b) $100 \mu \mathrm{m}$ and (c) $30 \mu \mathrm{m}$. Error bars, s.d. $\left(n=10 \mu \mathrm{MACs}\right.$ for each strain level, $\left.{ }^{* *} P<0.01,{ }^{* * *} P<0.001\right)$.

myotube differentiation in 3D. Myotubes with similar morphologies to state-of-the-art myotubes (myotubes induced from myoblasts cultured in 2D Petri dishes with horse serum) were evident in $\mu$ MACs strained to $40 \%$ (Figure 4). Differentiation of myoblasts in the strained $\mu$ MACs was verified using fluorescent staining and gene expression analysis of MyHC (Figure 4a) at day 10 of stretching as the standard measurement for myotube formation. In addition, the mRNA expression levels of the $\mathrm{MyHC}$ isoform ( $\mathrm{MyHC} \mathrm{IId} / \mathrm{x}$ ) were also significantly higher after culturing for 10 days. mRNA levels of Myod, Myogenin and
Mrf4, known transcriptional regulators of MyHC, also increased (Figures $4 \mathrm{~b}-\mathrm{e}$ ). Further studies on the effect of tensile strain on cell differentiation mechanisms (e.g., activation of RhoA) are still needed. Achieving these large strains is critical for characterizing such developmental phenomena. To demonstrate the maturation of differentiated myofibers in strained $\mu \mathrm{MACs}$, we characterized the structure and composition of $\mu \mathrm{MACs}$ after 4 weeks of culture. The $\mu$ MACs contained densely packed and aligned myofibers parallel to the direction of the applied strain (Figures $5 \mathrm{a}-\mathrm{c}$ ), an important 
a
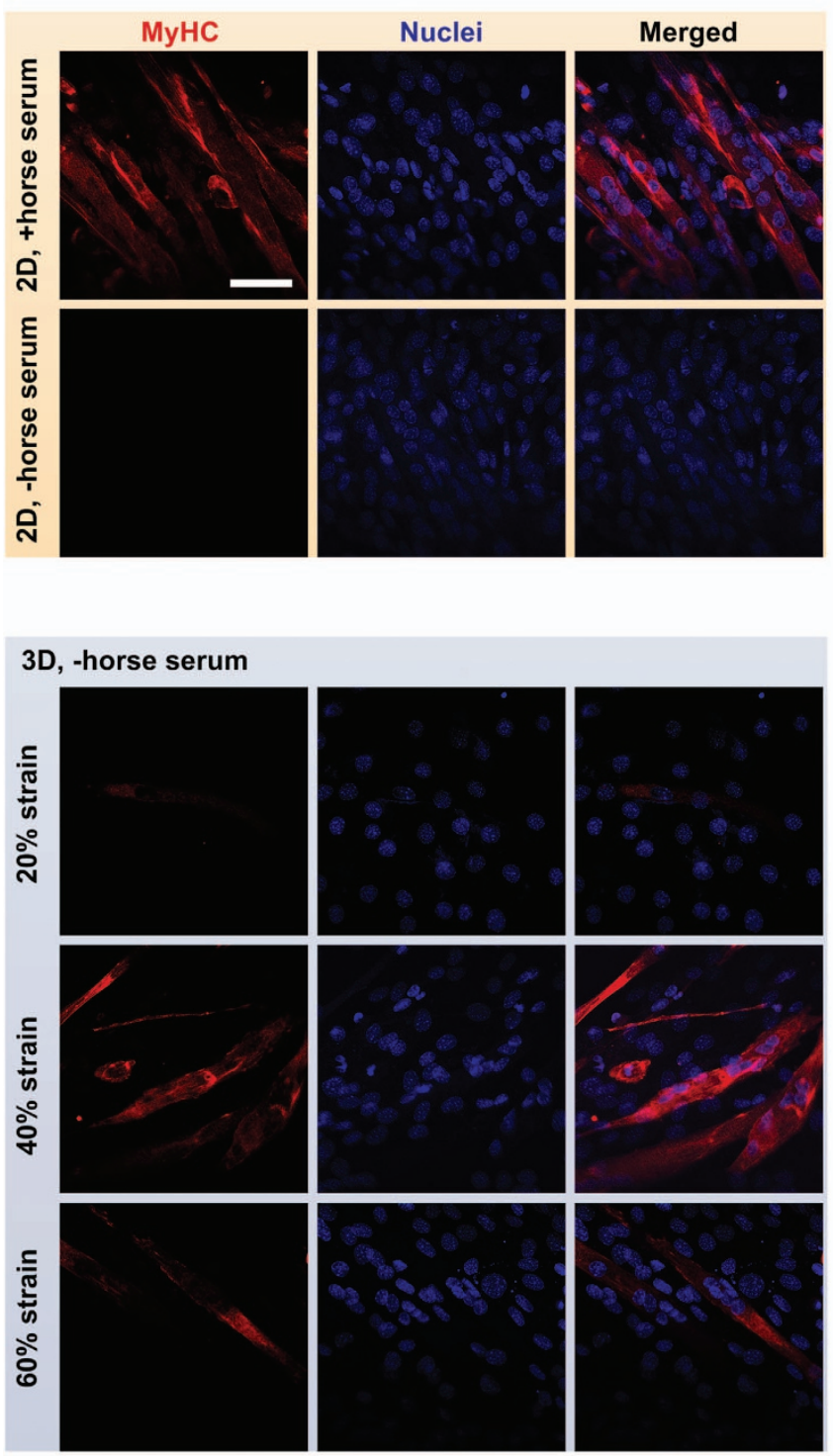

b
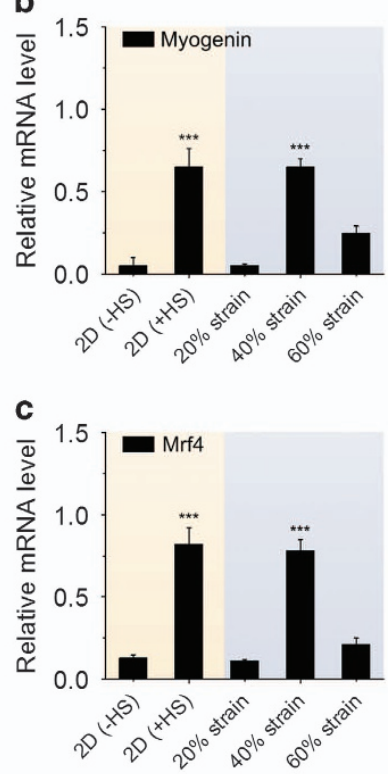

d
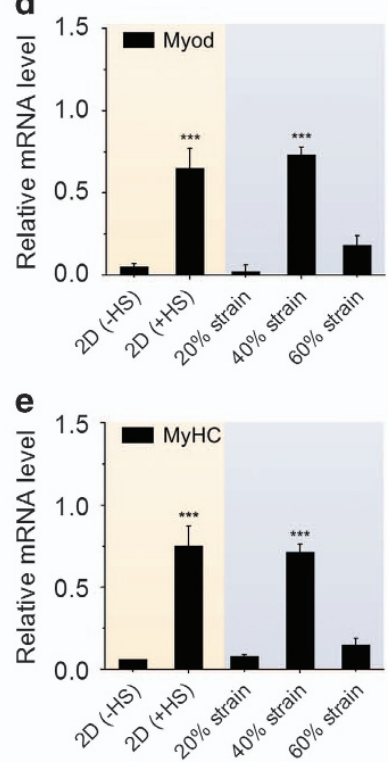

Figure 4 (a) Confocal fluorescence images of myosin heavy chain (MyHC) on a cover glass with horse serum (HS) and of strained microscale magnetically actuated, cell-laden hydrogels ( $\mu$ MACs) without horse serum at day 10 of culture. Formation of myotubes, a key step in myogenesis, was induced by straining C2C12 myoblasts cultured in $\mu \mathrm{MACs}$. (b-e) Myogenic gene expression was highest in three dimension (3D) after 40\% strain. Gelatin methacrylate (GelMA) modulus: $6 \mathrm{kPa}$. Scale bars: $50 \mu \mathrm{m}$. Error bars, s.d. ( $n=10 \mu \mathrm{MACs}$ for each strain level, $\left.{ }^{* *} P<0.001\right)$. 2D, two dimensional; 3D, two dimensional.

indicator of maturation. ${ }^{40}$ Structural maturation of myofibers over time was also evident from the progressive increase in myofiber diameter (from $16.5 \pm 1.7 \mu \mathrm{m}$ after 1 week of culture to $48.8 \pm 9.8 \mu \mathrm{m}$ after 4 weeks of culture) (Figure $5 \mathrm{~d}$ ) and from the expression of muscle-specific proteins including sarcomeric $\alpha$-actinin (SAA) and $\mathrm{MyHC}$ (Figure 5e and Supplementary Figure S11). Maturation of myotubes was also evident from confocal fluorescence images of transverse cross-sections of $\mu$ MACs, which showed striated patterns of SAA and uniformly distributed MyHC. These results suggest that the $\mu \mathrm{MAC}$ platform is a promising candidate for engineering of functional musculoskeletal tissues.

The degree to which strain, relative to other factors known to induce muscle myofiber differentiation such as engineering cell alignment through topographical patterns (e.g., grooves, pillars, and wrinkles) on 2D substrata, affected our observations is an important point to consider. ${ }^{3}$ We note that although $\mu$ MACs present cells with a uniform hydrogel ECM, straining provides both mechanical and steric directional cues to encapsulated cells, as the straining of the hydrogel induces anisotropy in the hydrogel microstructure that the cells may be able to sense. To ensure the suitability of $\mu$ MACs for long-term culture, degradation of the GelMA hydrogels was assessed because proteolytic enzymes (e.g., gelatinase and collagenases) secreted by encapsulated cells can degrade GelMA. We quantified the volumes of hydrogel samples (with residual liquid removed) using optical microscopy (Supplementary Figure S12a) and found a gradual $10 \%$ reduction in GelMA volume over the first 2weeks. However, the 
a
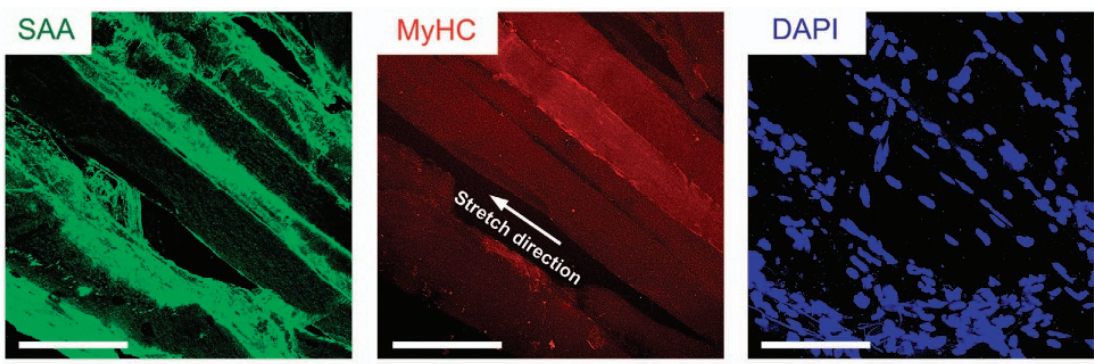

b
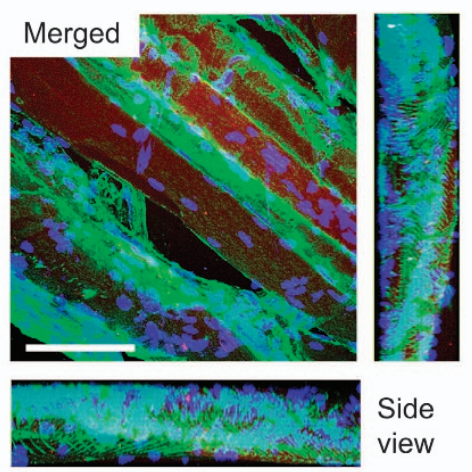

C
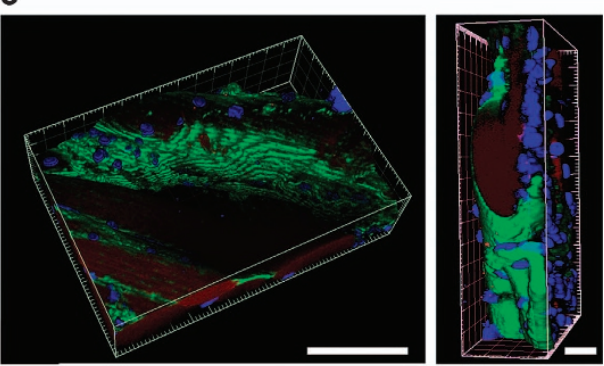

Side

view

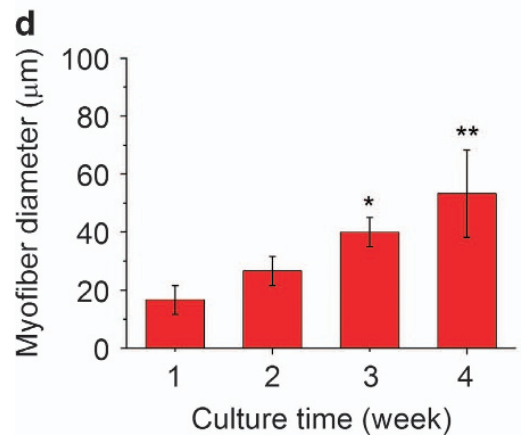

e

Culture time (weeks)

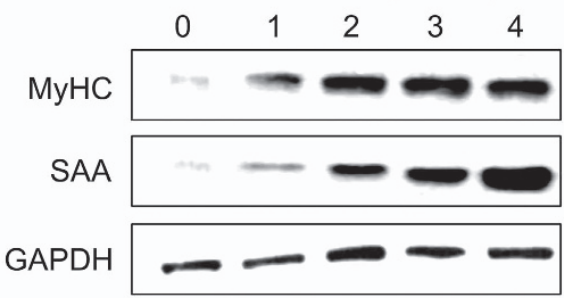

Figure 5 (a) Aligned myofibers within strained (40\%) microscale magnetically actuated, cell-laden hydrogels ( $\mu$ MACs) show uniformly distributed sarcomeric $\alpha$-actinin (SAA) and myosin heavy chain (MyHC) after 4 weeks of culture. (b) Merged confocal images of the side view of the $\mu$ MACs shows a striated pattern of SAA, indicating maturation of the myofibers. MyHC showed strong parallel alignment with the direction of tensile strain. (c) 3D reconstruction of confocal slices of matured myofibers in $\mu$ MACs. (d) Myofiber diameter increased with culture time. (e) Structural maturation of differentiated myofibers was also demonstrated from the increased expression of skeleton muscle markers including MyHC and SAA. $\mu$ MAC modulus: $6 \mathrm{kPa}$. Scale bars: (a, b) $100 \mu \mathrm{m}$, (c) $100 \mu \mathrm{m}$ (left) and $30 \mu \mathrm{m}$ (right). Error bars, s.d. ( $\left.n=15 \mu \mathrm{MACs},{ }^{*} P<0.05,{ }^{*} P<0.01\right)$. DAPI, 4',6-diamidino-2-phenylindole; GAPDH, glyceraldehyde-3phosphate dehydrogenase.

mechanical properties of cell-laden hydrogels cultured over a range of times appeared to be unaffected by this GelMA degradation (Supplementary Figure S12b).

\section{CONCLUSION}

In summary, $\mu$ MACs provide a useful platform for applying simultaneous, homogeneous, non-contact and well-controlled mechanical stimulation to cells in 3D culture. This platform overcomes challenges faced by existing 3D culture systems associated with sample handling, diffusion barriers, specimen variability and nonuniformity of mechanical loading. This system enabled the first-ever high strain testing of cells in a uniform 3D environment. Extension to the mechanobiological responses of different cell types, ECM compositions and tissue types is straightforward. $\mathrm{MACs}$ may be used as platforms for functional tissue engineering, drug screening and basic science, especially for cells at high strains. Overall, we believe that $\mu$ MACs will continue to be useful in the dynamic characterization of mechanobiological responses of cells in 3D, and, more generally, as a tool for non-contact mechanical characterization of soft materials.

\section{CONFLICT OF INTEREST}

The authors declare no conflict of interest.

\section{ACKNOWLEDGEMENTS}

This work was financially supported by the National Natural Science Foundation of China (11372243, 11522219 and 11532009), the Major International Joint Research Program of China (11120101002), the International Science and Technology Cooperation Program of China (2013DFG02930), the Research Fund for the Doctoral Program of Higher Education of China (20130201120071) and the China Postdoctoral Science Foundation (2013M540742). FX was also partially supported by the China Young 1000-Talent Program and Program for New Century Excellent Talents in University (NCET-12-0437). 
1 Fletcher, D. A. \& Mullins, R. D. Cell mechanics and the cytoskeleton. Nature 463 , 485-492 (2010).

2 Elson, E. L. \& Genin, G. M. The role of mechanics in actin stress fiber kinetics. Exp. Cell. Res. 319, 2490-2500 (2013).

3 Li, Y. H., Huang, G. Y., Zhang, X. H., Wang, L., Du, Y. A., Lu, T. J. \& Xu, F. Engineering cell alignment in vitro. Biotechnol. Adv. 32, 347-365 (2014).

4 Wang, L., Li, Y. H., Huang, G. Y., Zhang, X. H., Pingguan-Murphy, B., Gao, B., Lu, T. J. $\& \mathrm{Xu}, \mathrm{F}$. Hydrogel-based methods for engineering cellular microenvironment with spatiotemporal gradients. Crit. Rev. Biotechnol. 0, 1-13 (2015)

5 Cui, Y., Hameed, F. M., Yang, B., Lee, K., Pan, C. Q., Park, S. \& Sheetz, M. Cyclic stretching of soft substrates induces spreading and growth. Nat. Commun. 6, 6333 (2015).

6 Discher, D. E., Janmey, P. \& Wang, Y. L. Tissue cells feel and respond to the stiffness of their substrate. Science 310, 1139-1143 (2005).

7 Livne, A., Bouchbinder, E. \& Geiger, B. Cell reorientation under cyclic stretching. Nat Commun. 5, 3938 (2014).

8 Fu, J., Wang, Y. K., Yang, M. T., Desai, R. A., Yu, X., Liu, Z. \& Chen, C. S. Mechanical regulation of cell function with geometrically modulated elastomeric substrates. Nat. Meth. 7, 733-736 (2010).

9 Trepat, X., Wasserman, M. R., Angelini, T. E., Millet, E., Weitz, D. A., Butler, J. P. \& Fredberg, J. J. Physical forces during collective cell migration. Nat. Phys. 5, 426-430 (2009).

10 Cukierman, E., Pankov, R., Stevens, D. R. \& Yamada, K. M. Taking cell-matrix adhesions to the third dimension. Science 294, 1708-1712 (2001).

11 Bellas, E. \& Chen, C. S. Forms, forces, and stem cell fate. Curr. Opin. Cell. Biol. 31 92-97 (2014)

12 Hassan, W., Dong, Y. \& Wang, W. Encapsulation and 3D culture of human adipose derived stem cells in an in situ crosslinked hybrid hydrogel composed of PEG-based hyperbranched copolymer and hyaluronic acid. Stem. Cell. Res. Ther. 4, 32 (2013).

13 Legant, W. R., Pathak, A., Yang, M. T., Deshpande, V. S., McMeeking, R. M. \& Chen, C. $\mathrm{S}$. Microfabricated tissue gauges to measure and manipulate forces from 3D microtissues. Proc. Natl. Acad. Sci. USA 106, 10097-10102 (2009).

14 Legant, W. R., Miller, J. S., Blakely, B. L., Cohen, D. M., Genin, G. M. \& Chen, C. S Measurement of mechanical tractions exerted by cells in three-dimensional matrices. Nat. Meth. 7, 969-971 (2010).

15 Rodriguez, M. L., McGarry, P. J. \& Sniadecki, N. J. Review on cell mechanics: experimental and modeling approaches. Appl. Mech. Rev. 65, 1-41 (2013).

16 Wakatsuki, T., Kolodney, M. S., Zahalak, G. I. \& Elson, E. L. Cell mechanics studied by a reconstituted model tissue. Biophys. J. 79, 2353-2368 (2000).

17 Foolen, J., Deshpande, V. S., Kanters, F. M. \& Baaijens, F. P. The influence of matrix integrity on stress-fiber remodeling in 3D. Biomaterials 33, 7508-7518 (2012).

18 Sniadecki, N. J., Anguelouch, A., Yang, M. T., Lamb, C. M., Liu, Z., Kirschner, S. B., Liu, Y., Reich, D. H. \& Chen, C.S. Magnetic microposts as an approach to apply forces to living cells. Proc. Natl. Acad. Sci. USA 104, 14553-14558 (2007).

19 Zhao, R. G., Boudou, T., Wang, W. G., Chen, C. S. \& Reich, D. H. Decoupling cell and matrix mechanics in engineered microtissues using magnetically actuated microcantilevers. Adv. Mater. 25, 1699-1705 (2013).

20 Zhao, R. G., Chen, C. S. \& Reich, D. H. Force-driven evolution of mesoscale structure in engineered 3D microtissues and the modulation of tissue stiffening. Biomaterials $\mathbf{3 5}$, 5056-5064 (2014).

21 Li, Y. H., Poon, C. T., Li, M., Pingguan-Murphy, B., Lu, T. J. \& Xu, F. Chinese-noodleinspired muscle myofiber fabrication. Adv. Funct. Mater. 25, 5999-6008 (2015).

22 Krishnamurthy, G., Itoh, A., Bothe, W., Swanson, J. C., Kuhl, E., Karlsson, M., Craig Miller, D. \& Ingels, N. B. Jr. Stress-strain behavior of mitral valve leaflets in the beating ovine heart. J. Biomech. 42, 1909-1916 (2009).

23 Guccione, J. M., Costa, K. D. \& McCulloch, A. D. Finite element stress analysis of left ventricular mechanics in the beating dog heart. J. Biomech. 28, 1167-1177 (1995)

24 Scott, A., Khan, K. M., Heer, J., Cook, J. L., Lian, O. \& Duronio, V. High strain mechanical loading rapidly induces tendon apoptosis: an ex vivo rat tibialis anterior model. Br. J. Sports. Med. 39, e25 (2005).
25 Appleby-Thomas, G. J., Hazell, P. J., Sheldon, R. P., Stennett, C., Hameed, A. \& Wilgeroth, J. M. The high strain-rate behaviour of selected tissue analogues. J. Mech. Behav. Biomed. Mater. 33, 124-135 (2014).

26 Prevost, T. P., Balakrishnan, A., Suresh, S. \& Socrate, S. Biomechanics of brain tissue. Acta Biomater. 7, 83-95 (2011).

27 Cheng, F. -Y., Su, C. -H., Yang, Y. -S., Yeh, C. -S., Tsai, C. -Y., Wu, C. -L., Wu, M. -T. \& Shieh, D. -B. Characterization of aqueous dispersions of $\mathrm{Fe}_{3} \mathrm{O}_{4}$ nanoparticles and their biomedical applications. Biomaterials 26, 729-738 (2005).

28 Nichol, J. W., Koshy, S. T., Bae, H., Hwang, C. M., Yamanlar, S. \& Khademhosseini, A. Cell-laden microengineered gelatin methacrylate hydrogels. Biomaterials $\mathbf{3 1}$ 5536-5544 (2010).

29 Tai, H., Howard, D., Takae, S., Wang, W., Vermonden, T. \& Hennink, W. E. Photo-cross-linked hydrogels from thermoresponsive PEGMEMA-PPGMA-EGDMA copolymers containing multiple methacrylate groups: mechanical property, swelling, protein release, and cytotoxicity. Biomacromolecules 10, 2895-2903 (2009).

30 Fairbanks, B. D., Schwartz, M. P., Bowman, C. N. \& Anseth, K. S. Photoinitiated polymerization of PEG-diacrylate with lithium phenyl-2,4,6-trimethylbenzoylphosphinate: polymerization rate and cytocompatibility. Biomaterials $\mathbf{3 0}$, 6702-6707 (2009).

31 Schmittgen, T. D. \& Livak, K. J. Analyzing real-time PCR data by the comparative C (T) method. Nat. Protoc. 3, 1101-1108 (2008)

32 Xu, F., Inci, F., Mullick, O., Gurkan, U. A., Sung, Y., Kavaz, D., Li, B., Denkbas, E. B. \& Demirci, U. Release of magnetic nanoparticles from cell-encapsulating biodegradable nanobiomaterials. ACS Nano 6, 6640-6649 (2012).

33 Huang, G. Y., Zhang, X. H., Xiao, Z. P., Zhang, Q. C., Zhou, J. X., Xu, F. \& Lu, T. J. Cellencapsulating microfluidic hydrogels with enhanced mechanical stability. Soft Matter $\mathbf{8}$ 10687-10694 (2012)

34 Nakayama, A., Kakugo, A., Gong, J. P., Osada, Y., Takai, M., Erata, T. \& Kawano, S. High mechanical strength double-network hydrogel with bacterial cellulose. Adv. Funct. Mater. 14, 1124-1128 (2004).

35 Li, Y. H., Huang, G. Y., Zhang, X. H., Li, B. Q., Chen, Y. M., Lu, T. L., Lu, T. J. \& Xu, F. Magnetic hydrogels and their potential biomedical applications. Adv. Funct. Mater. 23, 660-672 (2013).

36 Schaffer, E., Norrelykke, S. F. \& Howard, J. Surface forces and drag coefficients of microspheres near a plane surface measured with optical tweezers. Langmuir 23, 3654-3665 (2007)

37 Lee, S. L., Nekouzadeh, A., Butler, B., Pryse, K. M., McConnaughey, W. B., Nathan, A. C. Legant, R. W., Schaefer, M. P., Pless, B. R., Elson, E. L. \& Genin, G. M. Physicallyinduced cytoskeleton remodeling of cells in three-dimensional culture. PloS ONE 7, e45512 (2012).

38 Yeh, J., Ling, Y., Karp, J. M. \& Khademhosseini, A. Micromolding of shape-controlled, harvestable cell-laden hydrogels. Biomaterials 27, 5391-5398 (2006).

$39 \mathrm{Du}$, Y., Lo, E. \& Khademhosseini, A. Directed assembly of cell-laden microgels for fabrication of 3D tissue constructs. Proc. Natl. Acad. Sci. USA 105, 9522-9527 (2008).

40 Madden, L., Juhas, M., Kraus, W. E., Truskey, G. A. \& Bursac, N. Bioengineered human myobundles mimic clinical responses of skeletal muscle to drugs. eLife 4 e04885 (2015)

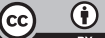

This work is licensed under a Creative Commons Attribution 4.0 International License. The images or other third party material in this article are included in the article's Creative Commons license, unless indicated otherwise in the credit line; if the material is not included under the Creative Commons license, users will need to obtain permission from the license holder to reproduce the material. To view a copy of this license, visit http:// creativecommons.org/licenses/by/4.0/

Supplementary Information accompanies the paper on the NPG Asia Materials website (http://www.nature.com/am) 